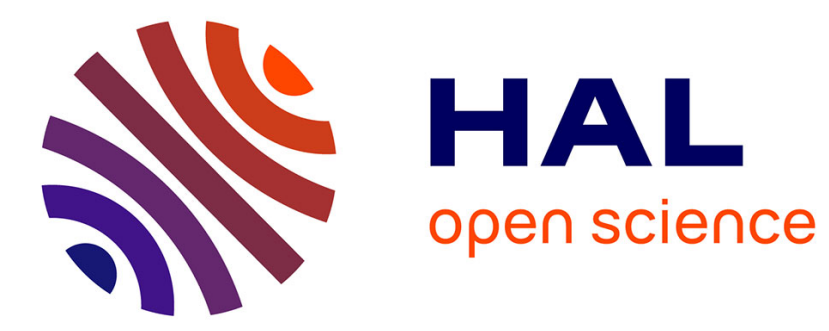

\title{
Le Pater breton de Vaudelin, une transcription phonologique en 1715
}

Erwan Le Pipec

\section{To cite this version:}

Erwan Le Pipec. Le Pater breton de Vaudelin, une transcription phonologique en 1715. Études celtiques, 2015, $\mathrm{n}^{\circ} 41$, pp.217-243. hal-02539583

\section{HAL Id: hal-02539583 \\ https://hal.science/hal-02539583}

Submitted on 10 Sep 2020

HAL is a multi-disciplinary open access archive for the deposit and dissemination of scientific research documents, whether they are published or not. The documents may come from teaching and research institutions in France or abroad, or from public or private research centers.
L'archive ouverte pluridisciplinaire HAL, est destinée au dépôt et à la diffusion de documents scientifiques de niveau recherche, publiés ou non, émanant des établissements d'enseignement et de recherche français ou étrangers, des laboratoires publics ou privés. 


\section{ÉTUDES \\ CELTIQUES}

FONDÉES PAR

J. VENDRYES

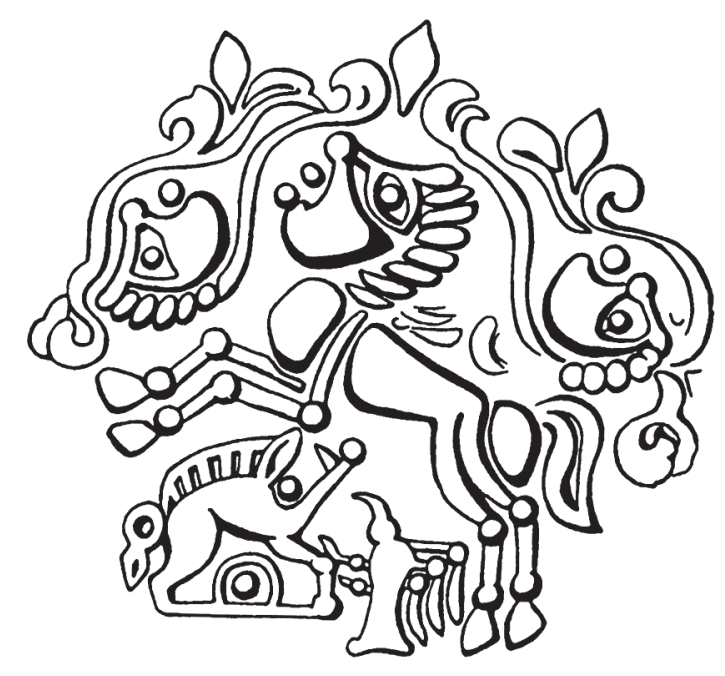

CNRS EDITIONS

15 rue Malebranche - 75005 Paris 


\section{ÉTUDES CELTIQUES}

Fondées par J. VENDRYES

Revue soutenue par l'Institut des sciences humaines et sociales du CNRS

\section{COMITÉ DE RÉDACTION}

Président :

Pierre-Yves LAMBERT

Président d'honneur :

Venceslas Kruta

Secrétaire :

Jean-Jacques CHARPY

Membres :

Brigitte FISCHER

Pierre FloberT

Patrick GaLliou

Hervé Le BiHaN

Jean LE Dô

Thierry LEJARS

Secrétaire de rédaction :

Marie-José LERoY

La rédaction remercie chaleureusement Christophe BAILLY

pour sa contribution à l'iconographie de ce volume.

Pour tout ce qui concerne la rédaction de la revue, s'adresser à

Pierre-Yves Lambert

212 rue de Vaugirard

75015 Paris

lambert.pierre.yves@gmail.com

et

Marie-José Leroy

Laboratoire Archéologie et Philologie d'Orient et d'Occident (CNRS-ENS)

marie-jose.leroy@ens.fr

Renseignements :

\section{CNRS ÉDITIONS}

15 rue Malebranche

75005 Paris

Tel. : 0153102700

Fax : 0153102727 


\section{LE PATER BRETON DE VAUDELIN : \\ UNE TRANSCRIPTION PHONOLOGIQUE EN 1715}

PAR

Erwan LE PIPEC

Le texte dont il est question ici est une version bretonne du Pater Noster, publiée en 1715 par le père Vaudelin. À première vue, rien d'extraordinaire, puisque l'on dispose pour cette époque de textes bien plus longs et bien moins normés, donc potentiellement plus instructifs pour l'histoire de la langue. Celui-ci est pourtant très original à bien des égards : d'une part, parce qu'il ne s'agit pas d'une traduction ou d'une adaptation comme tant d'autres, mais d'une transcription phonologique avant l'heure; d'autre part, en raison de quelques formulations très inhabituelles.

\section{La source}

Le texte se trouve dans un petit recueil, relativement bien connu des spécialistes de l'histoire du français ${ }^{1}$ : Instructions crétiennes mises en ortografe naturelle, pour faciliter au peuple la lecture de la science du salut, imprimé à Paris, par Jean-Baptiste Lamesle, en $1715^{2}$.

De son auteur, on ne sait quasiment rien. Marcel Cohen, qui a utilisé le livre pour étudier la prononciation française vers $1700^{3}$, ne fait que reprendre des informations soit données par l'auteur lui-même, soit présentes dans le privilège royal de son premier opus ${ }^{4}$. Le père Gile (ou Gilles ?) Vaudelin était donc un religieux des Augustins réformés de Paris, qui visiblement s'intéressait aux questions de prononciation et d'orthographe de son époque, puisqu’il mit au point en 1692 un système orthographique (en réalité un alphabet phonétique) destiné à simplifier la transcription du français. La même année, il l'adressa à l'Académie française, qui salua son travail, mais sans donner suite. En 1713, peut-être lassé d'attendre une reconnaissance qui ne venait

1. Brunot, 1966, t. VI, p. 948; Martinet, 1969, p. 155-167; Walter, 1976, p. 114; Chaurand, 1999, p. 279 et p. 337-338.

2. Le fac-similé est disponible en ligne, sur le site de Gallica (Bibliothèque nationale de France), dans l'édition de 1973 (Slatkine Reprints), où il est précédé de Nouvelle manière d'écrire comme on parle en France (1713) : http://gallica.bnf.fr/ark:/12148/bpt6k8433b. L'ouvrage de 1715 commence à la vue 47 .

3. Cohen, 1946.

4. Précisons toutefois que Cohen ne travaillait pas dans les meilleures conditions, puisqu'il rédigea son étude «comme dérivatif » en juin-juillet 1940, loin de sa bibliothèque, dans un coin de campagne des Deux-Sèvres où, sans doute, la débâcle l'avait mené. 
pas, il publia son système dans un opuscule : Nouvelle manière d'écrire comme on parle en France ${ }^{5}$, et en 1715, donc, ses Instructions crétiennes qui mettent en pratique ses principes, rebaptisés « orthographe naturelle».

Après des propos liminaires (préface expliquant le but de l'auteur et de son système; présentation du nouvel alphabet), le livre est très classique dans sa composition : il comprend (dans le désordre) un petit et un grand catéchisme sur le mode tridentin par questions et réponses ${ }^{6}$; les principales prières et formules rituelles en latin et en français; des «exercices spirituels ", dans la veine du temps; une table des fêtes mobiles et un calendrier liturgique; enfin, vient le corpus des oraisons latines, en orthographe classique. L'organisation de l'ouvrage est parfois déroutante, comme souvent dans ce type de document ancien, mais avec la complication des deux systèmes orthographiques, dont l'usage n'est pas constant, comme on l'a vu. Au milieu de l'ouvrage, à partir de la page 141, il propose une méthode «pour qui sachant lire l'orthographe naturelle, voudrait apprendre à lire le latin comme il est vulgairement orthographié et comme on le prononce aujourd'hui en France » (le tout rédigé en orthographe «naturelle»). Mais à partir de la page 150, il repasse à l'orthographe classique pour une nouvelle explication de la raison d'être de son système et de quelques règles de prononciation. Ces hésitations reflètent manifestement un projet mal défini : Vaudelin a voulu mettre en application son système, en transcrivant des textes à l'usage du «peuple», mais il n'a pu s'empêcher d'y glisser un manifeste, pour convaincre les déjà-lettrés du bien-fondé de sa réforme.

Ce qui nous intéresse vient à la page 156. Dans cette logique de défense et illustration de son alphabet, il entend en démontrer la validité en transcrivant plusieurs langues autres que le français. Et pour ce faire, s’inscrivant dans la tradition instaurée par Gessner ${ }^{7}$ au XVI ${ }^{\text {e }}$ siècle, il choisit d'orthographier le Pater Noster en trois langues mortes et en trois langues vivantes. Les langues mortes (outre le latin déjà présent) sont le grec, l'hébreu et le syriaque. Pour les langues vivantes, Vaudelin donne ses exemples en anglais, en allemand et, choix aussi inattendu que précieux pour le celtisant, en breton.

Cohen estime que «les textes en allemand et en anglais n'apprendront [...] que bien peu aux germanistes, tandis qu'il invite les historiens de la prononciation de l'hébreu et du syriaque à examiner les versions dans ces langues ${ }^{8}$ » (il était lui-même sémitologue de formation). En revanche, pas un mot sur le bas-breton. Il ne semble pas avoir pensé qu’il existait aussi des spécialistes des langues celtiques qui pouvaient être intéressés par cet extrait, ce qui explique peut-être pourquoi il est resté ignoré jusqu’à présent.

5. Voir, supra, note 2. L'opuscule compte 34 pages (correspondant aux vues 1 à 46 sur le site de Gallica).

6. Encore que ce procédé par questions et réponses soit une innovation protestante (voir Bisquerra, 2009).

7. GESSNER, 1555.

8. Cohen, 1946, p. 2. 


\section{Le texte}

\section{Le système de transcription}

La grande originalité, qui fait aussi la difficulté des écrits de Vaudelin, c'est que son orthographe naturelle est en réalité un alphabet spécifique. Celui-ci prend pour base l'alphabet latin, mais en modifie par quelques inflexions de nombreuses lettres, de façon à ce que chaque signe ne représente plus qu'un seul son. En cela, il est en avance de près de deux siècles sur son temps si l'on compare son projet à ceux de dialectologues et linguistes tels que l'abbé Rousselot ou Paul Passy. Mais à la différence des scientifiques de la fin du XIX ${ }^{\mathrm{e}}$ siècle, Vaudelin n’a pas conçu son système pour analyser la langue en laboratoire, mais pour la noter au quotidien : le double usage majuscule/minuscule est donc conservé. Par ailleurs, Vaudelin est tout de même enfant de son temps dans les présupposés théoriques qui guident son action : à la dernière page liminaire de la Nouvelle manière d'écrire, il présente un tableau assez complet qui expose sa conception de la langue et de l'orthographe. On y apprend que, pour lui, «la parole est un son articulé et choisi pour exprimer une pensée», ce qui ramène à la grammaire de Port-Royal, tandis que «l'orthographe est l'image de la parole», ce qui le situe à l'intérieur des débats orthographiques du français, qui opposent à l'époque comme aujourd'hui un courant étymologiste à une tendance phonétique, à laquelle Vaudelin se rattache.

La FIG. 1 reproduit le fac-similé de cet alphabet, tel qu'il apparaît dans la Nouvelle manière d'écrire .

\section{A LPHABET NOUVEAU.}

\section{a. an. ai. 6. in.i. e. o. on. ex. uss. on w. valews A. A. A. E. 1. 1. E. O. O. E. U. O. U. 2. 2. 2. e. i. i. e. o. p. द. u. o. u. \}figures.}

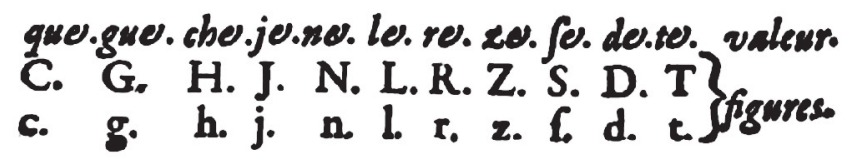

$$
\begin{aligned}
& \text { ve. fo. pe. be. mo. valews. } \\
& \text { V. F. P. B. M. } \\
& \text { v. f. p. b. m. sfowres }
\end{aligned}
$$

FIG. 1. L’alphabet présenté par Vaudelin dans sa Nouvelle manière d'écrire..., 1713 (@ BnF)

9. Vaudelin, 1713, p. 4. 
Alphabet phonétique donc, mais qui sert de base à un travail phonologique. Comme on le voit, Vaudelin confond signe et son minimal, que, bien sûr, il ne peut nommer «phonème", mais «son simple ${ }^{10}$ ». Ces «sons simples" sont implicitement considérés comme stables, sans variations allophoniques contextuelles, et comme des unités discrètes en nombre déterminé. Ainsi, Vaudelin fait plutôt œuvre de phonologue que de phonéticien lorsqu'il se sert du même graphème $<c>$ pour transcrire le /k/ des syllabes / ki/ et /ka/, soit $<c i>$ et $<\mathrm{ca}>$, bien que $/ \mathrm{k} /$ soit articulé de façon différente suivant qu'il est suivi d'une voyelle antérieure ou postérieure. Cette distinction n'étant pas pertinente dans le français de Vaudelin, elle n'est pas perçue, ni notée, et l'unité de /k/ l'emporte sur la variabilité des différents [k] possibles. Même constat devant l'utilisation de $<\mathrm{r}\rangle$, qui recouvre aussi bien les réalisations roulées, battues, grasseyées ou uvulaires (variation peut-être moins répandue à l'époque). En outre, les blancs typographiques sont respectés, ce qui, tout en étant un héritage de la tradition graphique, représente une mise en théorie de la langue et non un encodage brut ${ }^{11}$. L'utilisation des majuscules représente une autre entorse au principe phonétique, qui se justifie cette fois par des règles syntaxiques et sémantiques.

Mais dans son souci de pragmatisme, cette invariabilité des signes étant posée sur l'axe paradigmatique, Vaudelin utilise ceux-ci sur l'axe syntagmatique selon une règle d'occurrence qui est de nature phonétique. Si l'on prend ainsi l'exemple du mot -vous-, le principe phonologique induirait une transcription de type /vuz/, le /z/ final apparaissant systématiquement : sa réalisation ou sa non-réalisation à l'oral (sous la forme [z] de la liaison) dépend en effet de règles qui doivent être indiquées et qui doivent préciser les conditions de cette réalisation ou non-réalisation. Rien de tel chez Vaudelin, qui écrit $<$ vỎ $>$ ou $<$ vỏz $>^{12}$, au gré des circonstances, comme deux allomorphes, ainsi que le montrent les deux exemples de la FIG. $2^{13}$.

\begin{tabular}{|c|c|c|}
\hline Vaudelin & API & Orthographe standard \\
\hline je vó dmad pardo & [3ə vu dmãd pardõ] & Je vous demande pardon. \\
\hline je cra ce vóz at preza & [3ə kre kə vuz \&t prezã] & Je crois que vous êtes présent. \\
\hline
\end{tabular}

Fig. 2. Extraits et transcriptions de Vaudelin, 1715 (@ BnF).

Une telle transcription phonologique, qui réduit la réalité polymorphe de la langue à des prototypes invariants, n'est possible que parce que Vaudelin, en dépit du programme affiché, ne décrit pas le français tel qu'on le parle, mais tel qu'on devrait le parler. Il indique avoir « tasché de bien peindre la Prononciation qui est la plus usitée dans les conversations des gens de qualité qui parlent naturellement bien la Langue

10. Vaudelin, 1713, p. 2.

11. Vaudelin (1713, p. 11) en était conscient, puisqu'il écrit : «La Sagesse fait observer $1^{\circ}$ Que la Parole sort de la bouche si coulamment \& si serrément que toute une longue frase ne fait qu'un long Mot [...].»

12. Je transcris Vaudelin avec les polices actuellement disponibles.

13. VAudelin, 1715, p. 23. 
Françoise $^{14}$ », prononciation qui est par ailleurs «la plus usitée à la Cour, \& la seule Prononciation du beau Sexe ${ }^{15} »$. On remarquera au passage la contradiction : Vaudelin utilise le français de la Cour, tout en voulant instruire le peuple...

Il va sans dire que ce système, conçu pour un français très normé, était inadapté pour transcrire le breton. Cohen indique ${ }^{16}$ que Vaudelin n'a pas ajouté de signes pour rendre les sons inconnus en français, ce qui donne un résultat très curieux pour sa transcription de l'anglais, langue qu'apparemment il ignorait et qu'il a notée en piètre linguiste : les interdentales $[\theta]$ et [ð] sont notées $\langle\mathrm{t}\rangle$ et $\langle\mathrm{d}\rangle$, [i门] est noté grâce au signe qui lui sert à noter le [ẽ] français, les diphtongues sont réduites... Concernant le breton, les seuls sons qu'il ne pouvait directement transcrire sont les fricatives vélaires $[\mathrm{x}]$ et [y]. Pour y pallier, il écrit un $<\mathrm{r}>$ ou un $<\mathrm{c}>$ surmontés d'un signe diacritique ${ }^{17}$. Mais la grande lacune de l'«orthographe naturelle», c'est qu'elle ne note pas l'accent tonique, ni les longueurs vocaliques (à une exception près), bien que, sur ce dernier point, Vaudelin disposât de diacritiques pour le faire, qu'il a d'ailleurs utilisé en français ${ }^{18}$.

\section{Fac-similé et transcriptions}

Finalement, le lecteur a donc sous les yeux un texte assez curieux (Fig. 3).

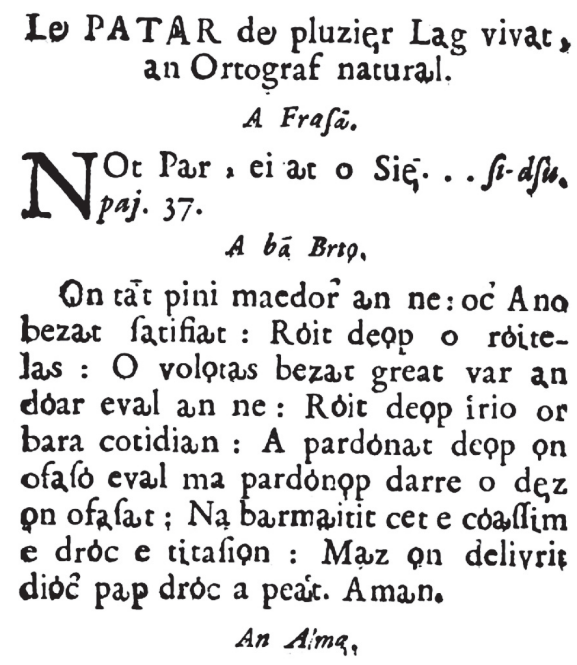

Fig. 3. Fac-similé du Pater, extrait de Vaudelin, 1715 (@ BnF).

14. Vaudelin, 1715 , p. 10.

15. Vaudelin, 1713, p. 28-29.

16. Cohen, 1946, p. 2.

17. Dans ses errata p. 247 , il signale qu'il faut ajouter «une virgule renversée» au-dessus des lettres concernées; pourtant, ce signe est déjà présent dans son texte.

18. Vaudelin, 1713, p. 11-13. 
Transposée en orthographes contemporaines des deux langues (étant donné le caractère inhabituel et peu commode du système), la page se lit ainsi :

Le PATER de plusieurs Langues vivantes,
en Orthographe naturelle.
En Français.
En bas-Breton.
otre Père, qui êtes aux cieux... ci-dessus, page. 37.
Hon tad pehini ma edoc'h en Neñv. hoc'h Anv
bezet sañtifiet. Roit deomp ho rouante-
lezh. Ho volontez bezet graet war an
douar evel en Neñv. Roit deomp hiriv hor
bara kotidien ha pardonit deomp hon
ofañsoù evel ma pardonomp d'ar re o deus
hon ofañset. Na bermetit ket e kouezhhemp
e droug e tañtasion, mez hon delivrit
diouc'h pep droug ha pec'hed. Amen.
En Allemand,

Si l'on transcrit le système de Vaudelin en alphabet phonétique international (API), on obtient du texte breton la version suivante (les citations discutées plus loin, qu'elles soient en orthographe moderne ou en API, feront référence à la numérotation des lignes utilisée ici) :
1) õn ta:t pini ma edoy $\varepsilon$ ne
2) oy ano bezet sãtifiet
3) ruit deõp o ruẽteles

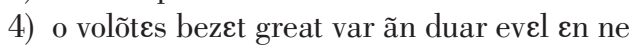
5) ruit deõp hirjo or bara kotidien
6) a pardunet deõp õn ofãsu
7) evعl ma pardunõp dar re o døz õn ofãset
8) na bermejtit ket e kwعsẽm e druk e tẽtasiõn
9) mez õn delivrit djux pep druk a pehet amen

Malgré ses imperfections, on a là un témoignage probablement unique de la prononciation du breton en 1715 . On relèvera qu'il y a vraisemblablement une erreur en 
2), où l'on conçoit mal que la voyelle initiale de <Ano> ne soit pas nasalisée (en l'occurrence, il manquerait un petit trait sous la barre centrale du A). Quelques corrections paraissent également nécessaires s'agissant de phénomènes de liaisons ${ }^{19}$. Bien sûr, la transcription est surtout très imparfaite du fait de l'absence d'informations prosodiques. Seul le mot/ta:t/ en 1) présente une indication de longueur. Détail qui ne gênera que le linguiste contemporain. Le lecteur bretonnant, tant au XxI qu'au $\mathrm{XVIII}^{\mathrm{e}}$ siècle, replacera facilement les accents là où il convient. Si l'on se fie aux témoignages des lexicographes et grammairiens des XVII ${ }^{\mathrm{e}}$ et $\mathrm{XVIII}^{\mathrm{e}}$ siècles, on peut hasarder que la prosodie du breton n'a que peu évolué depuis lors, ce qui permet de donner la transcription suivante :

\begin{tabular}{|c|}
\hline 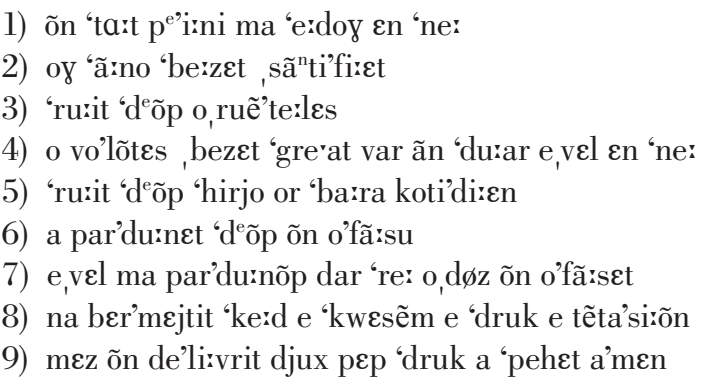 \\
\hline
\end{tabular}

\section{Commentaires}

\section{Contexte et cotexte}

Les celtisants avertis auront pu mesurer l'originalité de certaines formulations. Avant de pousser plus loin l'analyse, il me paraît nécessaire d'établir un corpus d'autres Pater qui fourniront une base de comparaison avec ce texte. En l'occurrence, ceux qui représentent un état de langue allant du moyen-breton au breton prémoderne. On dispose de plus d'une vingtaine de versions différentes du Pater grâce aux textes suivants :

1568 Heures de G. DE Kerampullh (quatrains en vers), édition StoKes, $1876^{20}$, p. 2 ;

1576 Catéchisme de G. De Kerampullh, éd. Stokes, $1876^{21}$, p. 58;

$1607^{22}$ Cosmographiae generalis, de P. Merula, fin du chapitre XV;

19. Il paraît ainsi douteux qu'un bretonnant ait prononcé [e 'druk e tẽta'sirõn], « dans le mal en tentation ", avec [k] suivi de [e]. On attendrait un adoucissement en ['drug]. D'autres liaisons théoriquement non-conformes sont attestées en parole naturelle.

20. Voir Kerampuilh, éd. Stokes, 1876.

21. Voir Kerampuilh, éd. Stokes, 1876.

22. Ce texte est signalé par Even, 1987. J'ai découvert après la rédaction du présent article qu'il ne s'agissait pas de l'édition originale, remontant elle à 1605 et dont le Pater présente quelques 
1610 Description [...] du Royaume de France, de F. DEs RuEs, p. 345 (copie du texte de Merula, avec quelques coquilles);

1622 Doctrin an christenien, de T. Gueguen (d'après Ledesme), éd. Hemon, $1977^{23}$, p. 18;

1625 Bellarmin, Declaration abundant eves an Catechism hac an doctrin Christen, trad. Gueguen, p. $231^{24}$;

1626 Dictionnaire et colloques français et breton de G. Quįuer, p. 17 (troisième

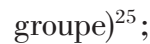

1631 Premier formulaire de prône en breton de Vannes, éd. LoTH, 1904;

1632 Dictionnaire et colloques français et breton de G. QuiQuer, $2^{\mathrm{e}}$ éd. augmentée (version nettement remaniée du Pater de 1626 p. 102 (troisième partie); $3^{\mathrm{e}}$ édition de 1633 : légères retouches, p. 66 (troisième partie);

v. 1660 An Abregé eus an doctrin christen, de J. MAUNOIR ${ }^{26}$, deux textes :

- 1660a, p. 7

- 1660b, p. 31;

1677 Doctrin an christenien, de T. Gueguen (d'après Ledesme), éd. Hemon, $1977^{27}$, p. 19 (texte de 1622 rajeuni et légèrement modifié);

1680 Doctrinal p. $50-51^{28}$;

1691 Catéchisme de Saint-Pol-de-Léon, cité par J. C. Adelung, 1809, t. II, p. 165;

1693 Second formulaire de prône en breton de Vannes, éd. Loth, 1890, p. 412-413;

1712 Heuriou brezounec ha latin, de Ch. LE BRIS, p. $1^{29}$. Ce texte est versifié, de sorte qu'il prend beaucoup de latitude par rapport aux versions canoniques ;

v. $1730^{30}$ Instruction var an Excellanç [...] ar Rosera, de Ch. LE BRIS, p. 198-202;

1775 Catechis imprimet dre urs an Autro [...] Escop ha Cont a Dreguer, p. 79;

1790 Instructioneu santel, du P. MARION, p. 464 (orthographe vannetaise);

1800 Grammatica latino-celtica, de A. Dumoulin, p. 178;

variantes graphiques très mineures (cf. édition de Nicolas de 1605, p. 432). Tout le travail de comparaison qui va suivre repose donc sur la version présente dans l'édition de Blaev de 1607, p. 88.

23. Voir Gueguen, éd. Hemon, 1977.

24. Je remercie H. Le Bihan de m'avoir transmis le texte du Pater que renferme cet ouvrage.

25. La pagination de l'édition de 1626 est irrégulière : elle va d'abord de 1 à 279, puis disparaît sur une quarantaine de pages, puis un troisième groupe de pages recommence de 1 à 79 . Ce découpage ne correspond pas tout à fait aux divisions du texte. Par ailleurs, l'orthographe du nom de l'auteur diverge d'une édition à l'autre : Quiquer ou Quicquer.

26. Voir Maunoir, 1707-1732 [1 ${ }^{\text {re }}$ éd. v. 1660].

27. Voir Gueguen, éd. Hemon, 1977.

28. Cité par Le Bihan, 2010. L'ouvrage présente de nombreux problèmes d'indentification (cf. LE BERRe, 2012, p. 230).

29. À défaut de l'original, j’ai utilisé l'édition de 1841 (voir LE BRIS, 1841).

30. Ce livre est daté de 1722 par J. Loth (1890, p. 339, note 1). La préface de l'ouvrage (p. xxvii) le situe toutefois sous le pontificat de Benô̂t XIII (1724-1730); p. xxx, une autre allusion le date après 1728. 
1807 Catechis evit an oll ilizou emeus an Impalaërder a Franç (catéchisme napoléonien de l'évêché de Quimper), p. 132;

1809 Plusieurs versions dans ADELUNG, 1809, dont une «dans un autre dialecte inconnu», tiré de Chamberlayne ${ }^{31}$, avec de nombreuses coquilles, t. II, p. 167 ;

1814 Catechis an escopty a Guemper (catéchisme de l'évêché de Quimper, édition de la Restauration, remplaçant le catéchisme napoléonien), p. 94;

1817 Catechis Treguer (catéchisme breton de Tréguier, réédition du catéchisme de 1783), p. 261 ;

1851 Catechim historik en abad Fleuri, trad. DréAno ${ }^{32}$, p. 89 (orthographe vannetaise).

Pour étoffer la base de données, on dispose aussi pour la même période de cantiques qui reprennent les paroles du Pater en les modifiant plus ou moins et en les mettant en musique. Ceux-ci figurent dans les recueils suivants :

1642 Cantiquou spirituel (anonyme), éd. Le Menn, 1997, p. 72-77 (2 cantiques);

1646 Canticou spirituel hac instructionou profitabl, de J. MaUnOIR, p. 63;

1712 Heuriou brezounec, de Ch. LE BRIS :

- Ar Pater noster, in Loth, 1890, p. 339,

- Cantic var ar pater noster, p. $516^{33}$;

v. 1730 Cantic var an orceson dominical, in Instruction var an Excellanç [...] ar Rosera, de Ch. LE BRIS, p. 441 ;

1734 Guaerzaenneu santél, in LoTh, 1890, p. 342 (orthographe vannetaise).

\section{Encodage/travail du transcripteur}

Beaucoup de questions entourent le travail de Vaudelin. Il indique que «ce sont des Naturels qui parlent bien leur langue» qui lui ont prononcé les Pater des langues vivantes, tandis que, pour les langues mortes, il s'est tourné vers des professeurs du Collège Royal de France ${ }^{34}$. Il n'est pas possible de savoir avec certitude à quelle époque il a noté ce Pater breton. Pour pouvoir présenter son système à l'Académie en 1692, il devait y travailler depuis la fin des années 1680. S'est-il essayé à le tester en notant le breton dès cette époque, ou a-t-il étendu son

31. Edward Chamberlayne est connu pour avoir publié en 1669 The Present State of England, réédité à partir de 1708 sous le titre The Present State of Great Britain. Il est possible que des versions du Pater soient mentionnées dans certaines éditions, pour illustrer les diverses langues celtiques (comme c'est le cas dans la Description générale de l'Univers de Salmon, de 1768 (SaLmon, trad. Jurain, 1776), où l'on trouve des Pater en une douzaine de langues, mais pas en breton). Malheureusement, sous ses deux titres, l'œuvre de Chamberlayne a connu trente-six éditions jusqu'à 1755. Je n'ai pas trouvé trace d'un Pater breton dans les versions que j’ai consultées.

32. Voir Fleury, trad. Dréano, 1851.

33. LE BRIS, 1841.

34. Vaudelin, 1715, p. 157. 
usage à mesure qu'il continuait ses recherches et en vue de l'ouvrage de 1715 ? On imagine en tout cas un paroissien ou un prêtre breton de Paris lui réciter sa prière, et lui, notant sous sa dictée. Les choses ne sont pourtant pas si simples, car il est clair que Vaudelin a aussi utilisé au moins une source écrite. Les blancs typographiques sont, comme pour le français, parfaitement respectés, sans erreur de coupe de mot, si ce n'est en 7), /d ar re/ orthographié en un seul mot <darre $>$. Pour le reste, tout s'accorde avec la tradition graphique du breton. Les coupes de mots sont même conformes à l'étymologie à la fin de la ligne 1), où la locution «aux Cieux» est transcrite -en Neñv- (orthographe moderne ${ }^{35}$ ), alors que l'erreur -en $E \tilde{n} v$ - avec fusion des deux /n/ de l'article et du nom était très fréquente chez les scribes bretons ${ }^{36}$.

On pourrait avancer que l'informateur savait écrire le breton et qu'il a contribué à la mise en forme du texte. Mais, au vu de l'originalité du système, il est peu probable que celui-ci ait pris une part active à la rédaction et plus vraisemblable que Vaudelin ait d'abord noté en orthographe classique, sous le contrôle de l'informateur breton, puis qu'il ait transcrit en s'appuyant conjointement sur l'écrit et l'oral.

Mais un point est particulièrement épineux. Comme on le verra en détail plus bas, le texte présente de nombreuses caractéristiques dialectales léonardes. Or le maintien de la coupe de mots -en Neñv- évoquée ci-dessus est à l'époque un phénomène typiquement vannetais. On ne le trouve que dans les versions du Pater de 1568 et 1576, puis dans les textes vannetais de 1693,1790 et $1851^{37}$.

Le père Vaudelin aurait-il utilisé un texte vannetais et entendu de vive voix un informateur léonard? C'est une possibilité, mais d'autres éléments viennent compliquer la situation.

35. Les transcriptions m'ont causé bien des hésitations, mais j’ai dû m’y résoudre dans les cas de figure suivants :

1. Le système de Vaudelin étant impossible à reproduire, il était exclu de le citer conformément à l'original. Seulement il était encore plus exclu de produire un artefact, «à la manière » des graphies de 1715. J'ai donc transcrit breton et français selon une norme contemporaine (et donc en l'occurrence en peurunvan, s'agissant du breton).

2. Étant donnée la grande instabilité graphique du breton entre 1568 et 1851, il n'était pas possible de citer chaque microvariation, encore moins quand je tenais précisément à dégager l'invariant, la permanence d'un prototype. Face à : en Eên, en Enff, en Eoûn, en nefuou, en Euffaou, etc., quelle graphie adopter pour en figurer l'étalon? À défaut de forme de référence, l'actuel en Neñ $v$ m'a paru être le choix le moins mauvais.

3. Quand un commentaire porte spécifiquement sur un point de phonétique, je donne mes exemples en API.

Sauf négligence de ma part, mes transcriptions obéissent à ces règles. Pour les autres cas de figure, quand je cite un texte d'époque, j'ai respecté la graphie d'origine.

36. C'est le cas par exemple dans les Pater de 1607, 1610, 1622, 1625, 1626, 1631, 1632, 1660, $1677,1680,1691,1712,1730,1775,1800,1807,1814$ et 1817.

37 . Il en va de même dans les cantiques : les recueils de 1642, 1646 et 1712, en orthographe de type léonard, présentent les formes -an eff-, -en Envou- et -en Eèvou-, tandis que le recueil vannetais de 1734 donne -énn nean-. Le prône vannetais de 1693 présente par ailleurs les deux formes. 


\section{Léonismes}

De nombreux détails indiquent une prononciation léonarde.

\section{Oralisation des voyelles nasales}

Pour les voyelles antérieures, ce n'est pas un phénomène spécifiquement léonard, puisqu'il concerne pratiquement toute la moitié ouest de la Basse-Bretagne, mais elle est plus fréquente au fur et à mesure que l'on progresse vers le nord-ouest, c'est-à-dire vers le Léon. On en voit des exemples dans :

- Neñv, «Cieux» (voir p. 222, l. 1 et 4), qui avait originellement une voyelle nasale, cf. vieux-breton nem, moyen-breton neff et eff (soit *[nẽw]); vannetais néan. La carte 161 du NALBB (idem) montre une concentration nette de formes de type [ne] dans le Léon.

- pardonit, «pardonnez» (l. 6), la voyelle [õ] évolue fréquemment en [u] dans le Léon, d'où [par'du:net].

\section{Fermeture des voyelles d'aperture intermédiaire}

- Roit, «donnez» (1. 3 et 5), /o/ étymologique devient /u/, d'où ['ruxit], vs. Roit ${ }^{38}$, Roït $^{39}$, Roet $^{40}$, Reit $^{41}$.

\section{Conservation et métathèse de la diphtongue /ae/}

- graet, «faite» (1. 4), est prononcé ['gre`at], à comparer à groet ${ }^{42}$, graet $^{43}$, grcet $^{44}$, gret $^{45}$, gret $^{46}$, grêt $^{47}$.

\section{Conservation du/z/ issu de l'interdentale /ð/}

- bezet, «soit» (1. 2 et 4), vs. beet et beèt $t^{48}$, beet ${ }^{49}$.

- volontez, «volonté» (1. 4), vs. bolonte ${ }^{50}$, polonte ${ }^{51}$.

38. Gueguen, 1622 et 1677 : voir Gueguen, éd. Hemon, 1977.

39. Le Bris, 1712.

40. Catechis an escopty a Guemper, 1814.

41. Catechis imprimet dre urs an Autro..., 1775.

42. Kerampullh, 1576 : voir Kerampuilh, éd. Stokes, 1876.

43. Merula, 1607.

44. Quiquer, 1632.

45. Gueguen, 1622 et 1677 : voir Gueguen, éd. Hemon, 1977.

46. Catechis imprimet dre urs an Autro..., 1775, et Catechis Treguer, 1817.

47. Catechis an escopty a Gumper, 1814.

48. Catechis imprimet dre urs an Autro..., 1775.

49. Catechis Treguer, 1817.

50. Dumoulin, 1800.

51. Catechis an escopty a Gumper, 1814. 


\section{Marques morphologiques}

- deomp, «à nous» (1. 3, 5 et 6), malgré une grande unité graphique, dans la langue parlée la double présence de la diphtongue /eo/ et du /p/ final n'est guère attestée que dans le Léon : $A L B B$, c. 109.

- bermetit, «permettez» (1. 8), delivrit, «délivrez» (1. 9), Roit, «donnez» (1. 3 et 5), présentent tous trois une désinence prononcée [it], typique du Léon : cf. $A L B B$, c. 352. Par comparaison : bermettet et delivret ${ }^{52}$, abandonet et dilivret ${ }^{53}$. On a en revanche une forme prononcée [ct] dans pardonit (l. 6).

\section{Phénomènes divers}

- pehini, «qui » (l. l) : on attendrait plutôt une prononciation en trois syllabes, du type [pe'ini]. La réduction à deux syllabes est attestée en prononciation familière, ce qui ne cadre pas avec la récitation de la prière, mais on la trouve (graphiée -pîni-) dans le Pater de Le Bris (1712), auteur léonard bien représentatif de son terroir linguistique.

- war, «sur» (l. 4), est prononcé [var]. Là encore, le phénomène n'est cependant pas limité au Léon. Par opposition : oar ${ }^{54}$; *aornomp, pour oarnomp, « sur nous ${ }^{55}$.

- rouañtelezh, «royaume» (1. 3), est prononcé [ruẽ’te:les]. La plupart des parlers bretons donnent pour ce mot une forme avec un [ã] à la place du [ẽ]. Toutefois, le Pater de 1680 présente Rouentelez, qui note vraisemblablement le même timbre. Cette évolution [ã] > [ẽ] est par ailleurs attestée pour d'autres mots en Léon.

- hiriv, «aujourd'hui » (1. 5), est prononcé ['hirjo]. Cette terminaison en [-jo] est assez caractéristique d'un grand quart nord-ouest de la Basse-Bretagne. Aujourd'hui, selon le $N A L B B$ (c. 96), cette prononciation est surtout attestée dans la vallée de l'Aulne, car le Léon lui a fait subir la syncope du /h/ initial. Jackson ${ }^{56}$ situe toutefois prudemment ce phénomène autour des XVII $-\mathrm{XVIII}{ }^{\mathrm{e}}$ siècles, en sorte qu'une prononciation léonarde ['hirjo] n'est pas invraisemblable en 1715.

- diouc'h, «à/de/contre» (1.9), se présente avec une évolution $/ \theta />/$ h/. Systématique en pays vannetais, celle-ci est aussi attestée sporadiquement en Léon ${ }^{57}$, même si l'écrit ne le reflète que partiellement : cf. Diouz an mintin, «le matin ${ }^{58}$ »; diouz e inclination, «selon son inclination ${ }^{59}$ »; Pedenno dious ar mintin ha dious an noz, «prière du matin et du soir ${ }^{60} » ;$ pellaat dioc'h ar goal gompagnunezou, «s'éloigner des mauvaises compagnies $^{61}{ } ;$ e hras mab dén doh é limage, «il fit l'homme à son image ${ }^{62}{ }$.

52. Catechis imprimet dre urs an Autro..., 1775, et Catechis Treguer, 1817.

53. Catechis an escopty a Guemper, 1814.

54. Catechis imprimet dre urs an Autro..., 1775.

55. Merula, 1607.

56. JACKSON, 1967, p. 561.

57. JACKSON, 1967, p. 531; FAGON, 2004.

58. Gueguen, 1622, p. 9 : voir Gueguen, éd. Hemon, 1977.

59. Le Bris, [v. 1710], p. 10.

60. Catechis imprimet dre urs an Autro..., 1775, p. 77.

61. Catechis an escopty a Guemper, 1814, p. 91.

62. Fleury, éd. Dréano, 1851, p. 1. 
Toutefois, ces nombreux traits dialectaux ne sont pas une garantie absolue que le Pater de Vaudelin lui ait été prononcé par un locuteur léonard. Aujourd'hui encore, chez les plus vieilles générations de bretonnants finistériens ou costarmoricains, une prononciation «à la léonarde» conserve un lustre et une solennité toute cléricale. Il n'est donc pas impossible que l'informateur ait récité un texte appris par cœur sous une forme très «léonisée ». Mais, à défaut de pouvoir juger ce qu’il en était il y a trois siècles, la présomption d'une origine léonarde du texte doit tout de même être considérée comme très forte.

\section{Hon Tad pehini ma edoc'h en Neñv...}

Le phénomène le plus remarquable du Pater de Vaudelin est cette formule introductive, version bretonne du "Notre Père qui êtes aux Cieux». Elle est extrêmement atypique, car l'usage courant pour des générations de bretonnants a toujours été : Hon Tad (pehini a) zo en Neñ $v^{63}$. Tous les Pater mentionnés plus haut montrent une traduction identique, ce qui fait de la version de Vaudelin un exemplaire unique.

Il y a deux grandes manières d'expliquer cette tournure : soit une reformulation endogène, soit une erreur par confusion. Selon la première hypothèse, la formule classique pehini zo aurait été ressentie comme une bizarrerie par un locuteur du XVIII ${ }^{\mathrm{e}}$ siècle. Cela, pour deux motifs. On peut en effet avancer que la formule de Vaudelin est plus conforme à la version latine, qui fournit le modèle à toutes les langues vernaculaires : dans Pater Noster qui es in Caelis, la morphologie verbale indique clairement la deuxième personne, le latin ne pouvant faire autrement ${ }^{64}$. En revanche, en breton, la forme verbale en zo (cf. supra) est une forme non marquée. Ce qui est grammaticalement correct, puisque le marquage morphologique du verbe n'apparaît qu'en l'absence du sujet et qu'il est systématiquement bloqué en sa présence (sauf cas particulier à la forme négative). Seulement les formes non marquées sont celles de la troisième personne du singulier. Donc, prise littéralement, la phrase Hon Tad pehini zo en Neñv peut se comprendre comme « Notre Père, qui *est aux Cieux ${ }^{65}{ }$. Si l'informateur de Vaudelin était latiniste, ou même francophone (ce qui est une quasi-certitude s'il se trouvait à Paris dans l'entourage du Père), il a pu ressentir la contradiction et a pu prendre l'initiative d'un marquage

63. Le pehini, qui traduit littéralement le pronom personnel qui du latin, considéré pour cette raison comme un calque incorrect, est tombé en désuétude vers le milieu du $\mathrm{xx}^{\mathrm{e}}$ siècle. Le $a$, particule verbale qui ne se traduit pas, se généralise au cours des XVII ${ }^{\mathrm{e}}$ et XVIII ${ }^{\mathrm{e}}$ siècles (voir WIDMER, 2012). Trois prototypes de formulation se sont donc succédé : Hon Tad pehini so en Neñv (XVI ${ }^{\mathrm{e}-}$

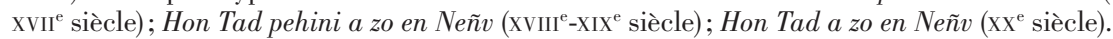

64. Laissons de côté la question du tutoiement et du vouvoiement. Le latin ne connaît que le tutoiement, les différents Pater bretons comme français ont utilisé les deux possibilités. Le texte de Vaudelin vouvoie; je ne baserai donc mon analyse que sur la deuxième personne du pluriel.

65. Tournure d'ailleurs courante en français parlé contemporain : «C'est moi qui est...», mais néanmoins incorrecte selon l'usage normatif, qui prescrit : «C'est moi qui suis... » L'anglais connaît la même opposition entre forme d'usage *It's me who is the tallest, théoriquement incorrecte, et forme prescriptive It's I who am the tallest, devenue désuète dans les faits. 
morphologique, que permettait la culture bretonnante de l'époque, comme on le verra plus loin.

Mais la gêne ressentie devant la formule consacrée peut aussi venir du pehini, qui brise l'unité et le mouvement de la phrase. Ainsi qu'il a été dit en note supra, ce pehini traduit littéralement le qui latin. Il n'est pas attesté dans la langue parlée et, pour cette raison, il est souvent considéré comme un calque transposé du latin et du français, depuis le Moyen Âge, par des traducteurs peu perspicaces. Que devrait-on alors avoir en lieu et place de pehini? Si l'on s'appuie sur le breton actuel, la deuxième personne du pluriel du verbe être s'exprime ainsi :

\begin{tabular}{|l|l|l|l|}
\hline \multirow{2}{*}{ Expression ordinaire } & \multirow{2}{*}{ Vous êtes } & Forme marquée & Oc'h \\
\cline { 3 - 4 } & & Forme non marquée & C'hwi zo \\
\hline Expression emphatique & \multirow{2}{*}{\begin{tabular}{l} 
Vu solennelle qui êtes \\
\cline { 3 - 4 }
\end{tabular}} & Forme autochtone & C'hwi a zo \\
\cline { 3 - 4 } & & Calque (?) & C'hwi pehini zo \\
\hline
\end{tabular}

Si pehini est vraiment un calque, alors l'expression autochtone a toujours présenté les mêmes traits, en 1715 comme aujourd'hui. La bonne traduction pour « Notre Père qui êtes...» est donc Hon Tad a zo... Bien sûr, l'usage du pehini était si répandu en chaire, qu'il devait être familier aux locuteurs. Mais il introduit une césure, que seule la virgule peut rendre en français :

\begin{tabular}{|l|l|}
\hline Hon Tad a zo... & Notre Père qui êtes... \\
\hline Hon Tad pehini zo... & Notre Père, qui êtes... \\
\hline
\end{tabular}

Cette césure ne pose pas de problème à un bretonnant empreint de culture classique, qui reconnaît derrière le masque du pehini le pronom de la grande langue, celle de la culture, de l'Église et de la doxa. Mais on peut penser qu'un bretonnant non lettré, qui se laisserait guider par le seul génie de la langue, serait conduit à réinterpréter la phrase et donc à la reformuler. Pehini coupe le lien entre Hon Tad/ «Notre Père» et le verbe $z o /$ «êtes». Lapostrophe, Hon Tad/ «Notre Père» devient donc une proposition averbale indépendante, tandis que pehini inaugure une proposition relative. Laquelle exige alors un verbe « être » conjugué indépendamment de Hon Tad/ « Notre Père ». S'enchaînent ensuite deux servitudes linguistiques : 1) le caractère aspectuel locatif de la phrase entraîne la forme locative du verbe être, soit (à l'époque) le radical $e d{ }^{66} ; 2$ ) une telle relative locative demande à être introduite par la conjonction $m a^{67}$, d'où Hon tad pehini ma edoc'h .

À l'appui de cette démonstration, il est possible de rapprocher du Pater de Vaudelin d'autres textes à peu près contemporains, qui montrent des formulations proches : en 1659, Maunoir, dans la grammaire bretonne annexée à son Sacré Collège de Jésus, donne les exemples suivants ${ }^{68}$, où l'on voit les mêmes structures de relatives locatives en $m a+e d$ - (notées ici $<i d->)$ :

66. Radical aujourd'hui sorti de l'usage, attesté par l'A $L B B$ uniquement sur l'île de Sein.

67. Favereau, 1997, p. 350.

68. MaUNOIR, 1659, p. 74. 
Ar Rouãtelez ma idi ar Sẽt

Elec'h ma idi ar marc'hat
Le Royaume où sont les Saints

Là où est le marché

Surtout, on dispose de la traduction de l'Oraison à son ange, présente dans les éditions de 1632 et 1633 des colloques français-breton de Quiquer, dont voici le texte in extenso ${ }^{69}$ :

Ael Doué $e^{70}$, pehiny maz ouch va Gardian, va conseruit, va diffennit ha va gouuernit

pecher paour mazouff me pehiny so

commettet dec dre an trugarez diuin euit dar

fin maz hilly assembles guenech ioüissaff

Rouantelez an euff ${ }^{71}$

On notera toutefois au passage que le verbe être utilisé ici n'est pas à la forme locative (prototype $o c^{\prime} h$ au lieu de $e d o c^{\prime} h$ ), preuve que les relatives introduites par $m a$ sont d'une classe plus large que les seules locatives (ce que confirme Favereau ${ }^{72}$ ). D'autre part à la troisième ligne, on relève pehiny so commettet dec (« qui suis commis à vous »), qui cohabite donc avec pehiny maz ouch ("qui êtes»). Mais il faut sans doute y voir une contrainte de style, puisque la phrase bretonne (légèrement différente de la phrase française) est :

va gouuernit pecher paour mazouff me pehiny so commettet dec...

gouvernez-moi, pauvre pécheur que je suis, qui suis commis à vous...

On a donc un enchaînement de deux relatives. L'utilisation de <pehiny so $>$ permet de les faire contraster et d'éviter la répétition un peu lourde qu'aurait été :

*pecher paour mazouff me pehiny mazouff commettet dec...

Une interrogation subsiste : les formes locatives à radical en ed- sont sorties d'usage assez précocement, au profit d'un radical en ema- (avec maintien dans le Léon, mais

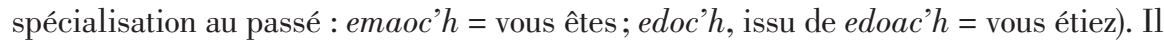
reste donc à savoir si, en 1715, le souvenir des formes verbales en ed-était encore assez vivant pour qu'un locuteur ait pu reformuler de lui-même «Notre père, qui êtes... » en Hon tad pehini ma edoc'h... en se conformant simplement au "génie de la langue", comme il a été dit. Cette hypothèse tendrait à être confirmée par le livre d'Euzen

69. Textes français et latin correspondants (QuiQuer, 1632, p. 102, troisième partie) :

Ange de Dieu qui estes mon Gardien, conseruez, deffendez \& gouuernez moy pauuvre pecheur qui suis cõmis à vous par la pieté supreme, à fin que ie puisse ensemble auecque vous ioüir du Royaume celeste.

70. Var. 1633 : Douè.

71. Var. 1633 : ioüissaff a Rouantelez an euff.

72. Favereau, 1997, p. 350.
Angele Dei qui custos es mei, pietate suprema, deffende \& guberna, ut valeam tecum cœlestia scandere regna. 
Roparz, An Imitation Jesus-Christ (L'imitation de Jésus-Christ), dont l'approbation est datée de 1689. Dans l'édition de Quimper de $1743^{73}$, on lit des phrases telles que :

da vont da leac'h ma edi ar joausted d'aller là où est la joie éternelle. eternel (p. 3)

equeit a ma edi e buhez (p. 22)

tant qu'il est en vie

E queit ha ma idomp er bet-man (p. 2l) Tant que nous sommes de ce monde

Manifestement, les formes en $e d$ - étaient donc encore bien usitées chez un auteur léonard au tournant des XVII ${ }^{\mathrm{e}}$ et XVIII ${ }^{\mathrm{e}}$ siècles. Le troisième exemple, ma idomp, montre que le paradigme était maîtrisé et que, même s'il n'était peut-être plus représenté dans la conversation quotidienne, il pouvait l'être dans une forme de discours élaborée.

Mais quand bien même cette explication de la divergence par les ressources internes de la langue serait juste, l'exceptionnalité du Pater de Vaudelin ne laisse pas de surprendre. La tournure pehini ma edoc'h a beau être grammaticalement acceptable, elle contraste décidément trop avec l'usage culturel de Hon Tad pehini zo en Neñv. Un peu comme si l'on avait en français : "Notre Père, qui vous trouvez aux Cieux $^{74}$ ». S'agissant d'un texte quelconque largement diffusé et identifié, comme une chanson populaire par exemple, une modification peut n'avoir qu'un effet parodique (procédé courant chez les imitateurs). Mais, quand la modification affecte un texte sacré, celle-ci a forcément une dimension transgressive, à moins qu'elle n'émane d'une autorité reconnue. Il est donc surprenant qu'un «Naturel parlant bien sa langue » ait pu prendre la liberté de retoucher ainsi le Pater. Une telle audace, qui plus est à une époque où la méfiance envers le jansénisme était paroxystique et le respect des formes prescrites scrupuleux, ne cadre pas avec le profil de l'informateur avancé plus haut. Celui-ci était peut-être un ecclésiastique, éventuellement membre de la même communauté que Vaudelin; à défaut, c'était au moins un paroissien fidèle, car on imagine mal Vaudelin se renseigner auprès du premier colporteur breton venu. Admettons toutefois que l'on n'ait pas affaire à un homme d'Église : s'il n'était pas léonard, il était tout de même singulièrement imprégné de culture religieuse pour pouvoir réciter un Pater très fortement marqué par la variante cléricale de prestige. Si l'on écarte cette imprégnation religieuse en avançant que les léonismes tiennent simplement à une origine effectivement léonarde, alors il faut convenir que sa maîtrise de la langue était fine, et relativement livresque, pour pouvoir fournir ce pehini ma edoc'h («qui êtes»). Dans tous les cas de figure que donne le croisement de ces données d'origines sociale et géographique, les pièces du puzzle ne s'assemblent pas : comment un tel informateur aurait-il pu aller contre l'usage unanimement admis?

73. ROPARZ, 1743.

74. On peut aussi penser aux paroles de la chanson Cendrillon du groupe Téléphone : «Notre Père, qui êtes si vieux...» Bien que la culture chrétienne n’imprégnât sans doute plus aussi fortement les jeunes Français de 1982 que les générations précédentes, le détournement, comme la source, sont évidents parce que le Notre Père reste une référence culturelle (un mème) à peu près universelle dans les sociétés européennes. 


\section{Flottements}

Il faut donc envisager une transcription erratique. Par là, il faut comprendre que Vaudelin (ou son informateur, mais c'est moins vraisemblable) aurait combiné différentes sources. D'autres éléments curieux du texte témoignent de la même manière d'une certaine imprécision :

- La ligne 8, pour «Ne nous soumets pas à la tentation", donne : Na bermetit ket e kouezhhemp e droug e tañtasion. Ce qui pourrait se traduire approximativement par : «Ne permettez pas que nous cédions au mal en tentation ${ }^{75}$ ». Approximativement, car cette apposition des mots $d r o u g / « m a l »$ et tañtasion/«tentation» est aux limites de la correction grammaticale et stylistique, aussi bien en breton qu'en français. Par ailleurs, ce doublet est là encore inhabituel. On ne le retrouve dans aucun des autres Pater, qui présentent toujours des variations sur les prototypes suivants ${ }^{76}$ (orthographes modernes) :

\begin{tabular}{|c|c|c|c|c|}
\hline \multirow[t]{3}{*}{1} & $\mathrm{a}$ & $\begin{array}{l}\text { Ha na zilez-ket ac'hanomp } \\
\text { en tañtasion. }\end{array}$ & $\begin{array}{l}\text { Et ne nous abandonne pas dans la } \\
\text { tentation. }\end{array}$ & 1568,1607 \\
\hline & $\mathrm{b}$ & $\begin{array}{l}\text { Ha n'hon abañdonit ket } \\
\text { d'an tañtasion. }\end{array}$ & Idem & 1807,1814 \\
\hline & c & $\begin{array}{l}\text { N'hon delaoskit ket en } \\
\text { tañtation. }\end{array}$ & $\begin{array}{l}\text { Idem (avec vouvoiement et sans } \\
\text { le « Et») }\end{array}$ & 1790,1851 \\
\hline \multirow[t]{4}{*}{2} & a & $\begin{array}{l}\text { Ha na gasit ket ac'hanomp } \\
\text { en tañtasion. }\end{array}$ & $\begin{array}{l}\text { Et ne nous conduisez pas en } \\
\text { tentation. }\end{array}$ & 1631,1693 \\
\hline & $\mathrm{b}$ & $\begin{array}{l}\text { Ha n'hon degasit ket e } \\
\text { tañtasion. }\end{array}$ & Idem & 1677,1809 \\
\hline & $\mathrm{c}$ & $\begin{array}{l}\text { Ha n'hon koñduzit ket en } \\
\text { tañtation }\end{array}$ & Idem & 1625 \\
\hline & d & $\begin{array}{l}\text { Ha na induizit ket ac'ha- } \\
\text { nomp en tañtasion. }\end{array}$ & Idem (ne nous induisez pas) & 1680 \\
\hline \multirow[t]{4}{*}{3} & a & $\begin{array}{l}\text { Ha na bermetit ket e } \\
\text { kouezhfemp en tañtasion. }\end{array}$ & $\begin{array}{l}\text { Et ne permettez pas que nous } \\
\text { tombions dans la tentation. }\end{array}$ & $\begin{array}{l}1622,1626, \\
1632,1633, \\
1730,1775, \\
1800,1817\end{array}$ \\
\hline & $\mathrm{b}$ & $\begin{array}{l}\text { Na bermetit ket e vemp } \\
\text { faezhet dre tañtasion ebet. }\end{array}$ & $\begin{array}{l}\text { Ne permettez pas que nous soyons } \\
\text { vaincus par la moindre tentation. }\end{array}$ & 1568,1660 \\
\hline & c & $\begin{array}{l}\text {... e vemp faezhet e nep } \\
\text { tañtasion }{ }^{77} \text {. }\end{array}$ & Idem & 1576 \\
\hline & d & $\begin{array}{l}\text { Ha na bermetit ket e vemp } \\
\text { trec'het dre tañtasion. }\end{array}$ & (Et) idem & 1691 \\
\hline
\end{tabular}

75. Littéralement : «que nous tombions dans le mal en tentation».

76. Pour les comparaisons introduites dorénavant, je n'utiliserai pas le texte de 1610, copie de celui de 1607.

77. Il s'agit d'une variante donnée par Stokes (Kerampullh, éd. StoKes, 1876), qui ne fournit pas le début de la phrase. 


\begin{tabular}{|cc|l|l|c|}
\hline 4 & a & $\begin{array}{l}\text { Ha n'hon lezit da gouezho } \\
\text { en tañtasion. }\end{array}$ & $\begin{array}{l}\text { Et ne nous laissez pas tomber } \\
\text { dans la tentation. }\end{array}$ & 1576 \\
\cline { 2 - 4 } & $\begin{array}{l}\text { N'hon lezit ket da } \\
\text { gouezhañ nepret, Gant nep } \\
\text { tañtasion ebet. }\end{array}$ & $\begin{array}{l}\text { Ne nous laissez jamais tomber } \\
\text { dans la moindre tentation. }\end{array}$ & 1712 \\
\hline
\end{tabular}

Cette classification tient compte de la formule d'apostrophe, mais on pourrait proposer un autre classement à partir du thème de la chute, de l'abandon ou de la défaite face à la tentation ${ }^{78}$. On remarquera que la formule la plus courante est celle présentée en $3 \mathrm{a}$, régulièrement attestée de 1622 à 1817. C'est par ailleurs celle que nous retrouvons chez Vaudelin, n'était cette adjonction de droug/«mal », alors que tous les Pater bretons dans la période délimitée n'utilisent que tañtasion.

En outre, l'erreur de prononciation [e 'druk e tẽta'sirõn], avec absence de liaison adoucissante à la jonction entre ['druk] et [e], accrédite l'idée que la séquence n'a pas été prononcée d'un trait, mais par à-coups.

- La ligne 9, pour Libera nos a malo/« délivrez-nous du mal», donne également une formule redondante : Mes hon delivrit diouc'h pep droug ha pec'hed, soit littéralement : «délivrez-nous de tout mal et péché ». Là encore, aucun autre Pater breton ne donne une telle formulation. La traduction la plus fidèle de la version latine appellerait plutôt le mot droug et c'est bien celui qui est très majoritairement utilisé ${ }^{79}$ :

\begin{tabular}{|c|c|}
\hline Droug & Pec'hed \\
\hline 1576,1607, & 1622, \\
1625, & 1626, \\
$1631,1633,1660$ (a et b), & \\
$1677,1680,1691,1693$, & \\
$1712,1730,1775,1790$, & \\
$1800,1807,1809,1814$, & \\
1817,1851 & \\
\hline
\end{tabular}

Seuls Gueguen et Quiquer utilisent donc le mot pec'hed, et encore, les rééditions de Quiquer postérieures à 1626 rétablissent droug ${ }^{80}$. En tout cas, comme on

78. Les recueils de cantiques exploitent les mêmes canevas. Chez LE BRIs, [v. 1730], on trouve en plus le thème du consentement à la tentation (qui ne doit pas être permis).

79. Le Pater de 1568 étant composé de quatrains en vers, il prend des libertés avec les formules canoniques. Pour la phrase concernée, il donne :

Hoguen hon diliuret dre ho puissancc

uez hon oll anquen ha souffrancc

a liammou hon aduerser

hon groet exempt e pep amser.
Mais délivrez-nous par votre puissance,

De toute notre angoisse et souffrance.

Des liens de notre Adversaire,

Libérez-nous en tout temps.

80. Dans l'édition de 1632, la phrase est coupée avant le mot fatidique... 
le voit, les Pater se répartissent l'usage de l'un ou l'autre mot, mais sans jamais juxtaposer les deux ${ }^{81}$.

\section{À la recherche du texte perdu...}

Du croisement des deux tableaux précédents, il ne faudrait pas conclure trop vite que Vaudelin a eu sous les yeux le Dictionnaire et colloques... de Quiquer, et qu'il y a trouvé son texte du Pater. Il est vrai que l'ouvrage devait pouvoir se trouver à Paris, puisqu'il consiste en un phrasebook destiné entre autres aux voyageurs. Mais le Pater de Vaudelin présente trop de divergences, en d'autres endroits, avec ceux de 1622 et 1626 , comme on va le voir.

Le texte du Pater Noster étant très court, il présente l'avantage de permettre une vue d'ensemble assez rapide et donc des comparaisons aisées. Sans examiner de trop près tous les détails, il est peu coûteux de mettre au point des grilles d'analyse phrase par phrase (parfois en zoomant sur tel membre de phrase), permettant de pointer convergences et divergences. On obtient alors les résultats suivants :

\begin{tabular}{|c|c|}
\hline $\begin{array}{l}\text { Hon Tad pehini ma } \\
\text { edoc'h en Neñv, }\end{array}$ & $\begin{array}{l}\text { «Notre Père, qui êtes aux Cieux,» } \\
\text { Tous les autres Pater divergent. }\end{array}$ \\
\hline $\begin{array}{l}\text { Hoc'h Anv bezet } \\
\text { sañtifiet. }\end{array}$ & $\begin{array}{l}\text { "Que votre nom soit sanctifié.» } \\
\text { La phrase est assez constante. Au lieu de bezet, «soit», les textes de } \\
1622 \text { et } 1626 \text { donnent da vet; les Pater vannetais de } 1790 \text { et } 1851 \\
\text { donnent re-vou; celui de } 1712 \text {, ra vezo. }\end{array}$ \\
\hline $\begin{array}{l}\text { Roit deomp ho } \\
\text { rouantelezh. }\end{array}$ & $\begin{array}{l}\text { «Donnez-nous votre Royaume. " } \\
\text { La formule n'apparaît qu'en } 1660 \text { (a et b), reprise en 1680, puis dans } \\
\text { les trois textes quimpérois de } 1800,1807 \text { et } 1814 \text {. Les autres textes } \\
\text { utilisent le subjonctif, avec prédominance du verbe en tête chez les } \\
\text { plus anciens : Deuet ho rouantelez, "Vienne votre Royaume» (1576), } \\
\text { puis du sujet en tête à partir du XVII }{ }^{\mathrm{e}} \text { siècle : Ho rouantelez deuet demp, } \\
\text { «Votre Royaume nous vienne» (1632). }\end{array}$ \\
\hline $\begin{array}{l}\text { Ho volontez bezet } \\
\text { graet }\end{array}$ & $\begin{array}{l}\text { "Que votre volonté soit faite» } \\
\text { Les Pater sont à peu près unanimes. Le texte de } 1607 \text { utilise eol pour } \\
\text { volonté; le texte de } 1680 \text { place le verbe (bezet gret) en tête; les textes } \\
\text { vannetais de } 1790 \text { et } 1851 \text { donnent encore une fois re-vou et celui } \\
\text { (léonard) de } 1712 \text {, ra vezo. }\end{array}$ \\
\hline $\begin{array}{l}\text { war an douar evel } \\
\text { en Neñv. }\end{array}$ & $\begin{array}{l}\text { «sur la terre comme au Ciel.» } \\
\text { Cette formule apparaît en 1625, puis en 1677. Elle devient régulière } \\
\text { à partir de } 1712 \text {, sauf dans les textes quimpérois }(1800,1807,1814) \text {. } \\
\text { Ces trois derniers lui préfèrent la formule plus ancienne : en douar euel } \\
\text { en euff (1632), ou même quen en eun quen en douar (1693). L'équivalent } \\
\text { en français : «en la terre», a été utilisé jusqu'au XVIII" siècle. }\end{array}$ \\
\hline
\end{tabular}

81. Les cantiques utilisent également tous le mot droug, sauf le deuxième cantique de 1642 (Cantiquou spirituel, éd. Le Menn, 1997, p. 80), qui donne goual, «malheur», sorti de l'usage comme substantif, mais conservé comme préfixe. 


\begin{tabular}{|c|c|}
\hline $\begin{array}{l}\text { Roit deomp hiriv } \\
\text { hor bara kotidien }\end{array}$ & $\begin{array}{l}\text { «Donnez-nous aujourd'hui notre pain quotidien» } \\
\text { Le trait saillant est ici l'emprunt au français, kotidien. Il n’apparaît qu'en } \\
1622 \text { et } 1626 \text {. Tous les autres textes utilisent pemdezyec (ici : 1576). }\end{array}$ \\
\hline $\begin{array}{l}\text { ha pardonit deomp } \\
\text { hon ofañsoù }\end{array}$ & $\begin{array}{l}\text { «et pardonnez-nous nos offenses» } \\
\text { Formulation quasi unanime (le texte de } 1607 \text { dit pechedou, «péchés» } \\
\text { au lieu d'offenses; celui de } 1712 \text { reformule pour la métrique). }\end{array}$ \\
\hline $\begin{array}{l}\text { evel ma pardonomp } \\
\text { d'ar re }\end{array}$ & $\begin{array}{l}\text { «comme nous pardonnons à ceux » } \\
\text { Toutes les versions antérieures à 1632, puis celles de 1730, } 1807 \text { et } \\
1814 \text { utilisent le singulier (d'an nep). }\end{array}$ \\
\hline o deus & $\begin{array}{l}\text { "qui nous ont offensés.» } \\
\text { Les Pater varient peu. Celui de } 1607 \text { donne da nep pegant ezomp } \\
\text { offanczet, "à celui par qui nous sommes offensés»; ceux de 1622, } \\
1626,1632 \text { et } 1633 \text { présentent le prototype : o deus ni ofañset, dont } \\
\text { la correction pose question; en 1691, le pronom «qui » est traduit par } \\
\text { le calque pere. }\end{array}$ \\
\hline$N a b$ & $\begin{array}{l}\text { «Ne permettez pas» } \\
\text { Le sens de la phrase latine Ne nos inducas fait polémique depuis } \\
\text { deux mille ans : le mal ne pouvant venir de Dieu, comment peut-Il } \\
\text { «conduire vers» la tentation? Il s'en est suivi une abondante littérature } \\
\text { exégétique pour interpréter le texte en comparant le latin, le grec, } \\
\text { l'araméen et l'hébreu. Cette formulation « Ne permettez pas» remonte } \\
\text { à un commentaire de saint Augustin. On la retrouve en plusieurs } \\
\text { langues, à différentes époques : en français, elle aurait été incluse } \\
\text { dans le Pater en } 1507 \text {. C'est sans doute aux jésuites, grands diffuseurs } \\
\text { de recommandations didactiques sur la prière, que l'on doit son } \\
\text { introduction en breton. Elle apparaît dans les Pater de } 1568,1622, \\
\text { 1626, 1632, 1633, 1660, 1691, 1730, 1775, 1800 et } 1817 \text { (cf. supra). }\end{array}$ \\
\hline e kouezhhemp & $\begin{array}{l}\text { «que nous tombions» } \\
\text { Le verbe «tomber» est étroitement lié à la séquence précédente. } \\
\text { Seuls les textes de } 1568,1576,1660 \text { et } 1691 \text { découplent leur usage en } \\
\text { disant : «Ne permettez pas que nous soyons vaincus par...» (faezet/ } \\
\text { trec'het). La prononciation [s] (ici associée aux graphèmes <zhh>) est } \\
\text { un archaïsme, que l'on retrouve jusqu'en } 1817 \text { (noté <coësemp>). Elle } \\
\text { résulte du contact entre la base verbale kouezh- et l'ancien morphème } \\
\text { de conditionnel -he, aujourd'hui -fe. }\end{array}$ \\
\hline $\begin{array}{l}\text { e droug e tañta- } \\
\text { sion, }\end{array}$ & \begin{tabular}{|l} 
«dans le mal en tentation,» \\
Tous les autres Pater divergent (cf. supra).
\end{tabular} \\
\hline mez hon delivrit & $\begin{array}{l}\text { «Mais délivrez-nous» } \\
\text { Il y a une séparation assez nette entre textes anciens et modernes : } \\
\text { avant 1691, le mot «mais» est traduit par hoguen. Après cette date, } \\
\text { par moes. Seules exceptions à cette règle : les textes de 1631, puis } 1800 \\
\text { et 1809. Par ailleurs, les textes vannetais de } 1790 \text { et } 1851 \text { utilisent } \\
\text { delivrit-ni (de même sens). }\end{array}$ \\
\hline diouc'h & quement en 1607 (dyouz) et 1680 (dious). \\
\hline
\end{tabular}




\begin{tabular}{|l|l|}
\hline pep droug & $\begin{array}{l}\text { «tout mal » } \\
\text { Le mot pep, tout, n'est présent qu'en 1660a, } 1680 \text { et 1775. Les autres } \\
\text { Pater oscillent entre a zrouc, eus an drouc, ag en droug (de même sens). }\end{array}$ \\
\hline ha pec'hed. & $\begin{array}{l}\text { «et péché. » } \\
\text { Présent uniquement en } 1622 \text { et 1626, au lieu de droug (cf. supra). }\end{array}$ \\
\hline
\end{tabular}

Le Pater de Vaudelin ne correspond donc rigoureusement à aucune autre version disponible pour la période retenue. Dans l'ensemble, il présente des caractères de modernité, qui le rattachent assez clairement à la deuxième moitié de cette période. Mais on y retrouve aussi en divers endroits des archaïsmes ou des spécificités. Comme s'il s'agissait d'un patchwork de tous les Pater qui pouvaient se réciter en Basse-Bretagne. Il est toutefois possible d'affiner cette impression et de faire des rapprochements plus étroits. Par exemple, à partir du découpage séquentiel du tableau précédent, en faisant la somme des points de convergence et de divergence entre le Pater de Vaudelin et les autres. N'ont été prises en considération que les différences fondamentales de formulation, affectant la structure de la phrase ou une valeur sémantique : dans evel ma pardonomp d'ar re/ «comme nous pardonnons à ceux", le texte de 1631, eual ma pardonnamp den nep/ «comme nous pardonnons à celui », présente une divergence par l'emploi du singulier au lieu du pluriel. En revanche, ne sont pas considérées comme divergences les variations de surface, telles l'orthographe (evel = eual; pehiny = pehini $;$ eus = «eux », etc.) ou l'adaptation à une prononciation locale $($ pardonomp $=$ pardonnamp $;$ deomp $=$ dimp $;$ offançou $=$ offanseu $=$ offanso $)$. Je n'ai pas non plus retenu les petites variations de synonymes (evel $=e$-giz, comme), ni l'ajout ou l'omission de $h a$, «et». On obtient alors le classement suivant, figurant l'ordre croissant de proximité avec le texte de Vaudelin ${ }^{82}$ :

\begin{tabular}{|c|c|c|c|c|c|c|c|c|c|c|c|c|}
\hline & $\begin{array}{l}\text { 건 } \\
\text { نै }\end{array}$ & $\stackrel{\varpi}{v}$ & गे & $\stackrel{\infty}{\circ}$ & $\overrightarrow{\widehat{a}}$ & $\vec{g}$ & $\begin{array}{l}\bar{a} \\
\stackrel{0}{0}\end{array}$ & $\overrightarrow{\mathfrak{d}}$ & $\underset{ \pm}{\infty}$ & 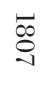 & a & $\overbrace{0}^{\infty}$ \\
\hline Convergences & 10 & 9 & 8 & 8 & 8 & 7 & 7 & 6 & 6 & 6 & 6 & 6 \\
\hline Divergences & 6 & 7 & 8 & 8 & 9 & 9 & 10 & 9 & 10 & 10 & 10 & 10 \\
\hline Écart & +4 & +2 & 0 & 0 & -1 & -2 & -3 & -3 & -4 & -4 & -4 & -4 \\
\hline
\end{tabular}

\begin{tabular}{|c|c|c|c|c|c|c|c|c|c|c|c|c|}
\hline & $\widehat{⿶}$ & 怘 & 命 & 矛 & 占 & 苓 & 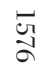 & 키 & $\underset{\substack{\infty \\
\stackrel{\infty}{i}}}{ت}$ & 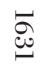 & $\vec{g}$ & $\vec{J}$ \\
\hline Convergences & 6 & 6 & 6 & 6 & 6 & 5 & 5 & 5 & 5 & 5 & 2 & 1 \\
\hline Divergences & 10 & 12 & 12 & 12 & 12 & 11 & 11 & 12 & 12 & 13 & 17 & 19 \\
\hline Écart & -4 & -6 & -6 & -6 & -6 & -6 & -6 & -7 & -7 & -8 & -16 & -18 \\
\hline
\end{tabular}

Les deux échelles, de convergence et de divergence, sont à peu près symétriques et donnent un différentiel régulier. C'est donc le Pater de 1775 qui est le plus proche

82. Le texte de 1568 , recomposé en quatrains, n'a pas été retenu, non plus que celui de 1610 (cf. supra). 
de Vaudelin, et celui de Le Bris de 1712 qui en est le plus éloigné. Ce résultat peut surprendre, puisque le Pater de Le Bris est paradoxalement le texte qui est le plus proche de Vaudelin dans le temps et sans doute aussi dans l'espace (le Léon). Mais cela tient au fait que ce Pater est en vers et qu'il reformule parfois hardiment le Pater canonique, comme on l'a déjà dit (l'autre texte de Le Bris, celui de 1730, est d'ailleurs beaucoup mieux placé). À ce détail près, la position des textes les plus éloignés s'explique facilement : il s'agit des textes vannetais (1631, 1851, 1790, 1693) ou anciens $(1576,1625,1632,1626,1622,1633)$. Le Pater de 1607 concentre plusieurs critères de marginalité : il est assez ancien; il pourrait avoir été produit dans le Trégor (cf. *aornomp, pour oarnomp, «sur nous», avec [w] initial); il serait de source protestante, comme en attesterait la doxologie ${ }^{83}$. À l'inverse, les textes qui se situent à un niveau plus proche, bien qu'étant étroitement imbriqués, semblent pouvoir se rattacher à deux courants. Il y a d'une part ceux qui ont été produits au sortir du moyen-breton, qui présentent les premiers infléchissements vers une graphie dialectale léonarde (une sorte de «pré-léonard») : c'est le cas du catéchisme de SaintPol-de-Léon $(1691)^{84}$, de celui du Doctrinal (1680), de ceux du père Maunoir, apôtre de la Contre-Réforme (1660, peut-être aussi 1677). Et d'autre part les textes plus récents, produits dans la région de Quimper (1807 et 1814 ${ }^{85}$, 1800). En dépit de leur date d'impression, ceux-ci sont légèrement archaïsants et contextuellement marqués par l'empreinte de l'Ancien Régime ${ }^{86}$. Pour se rapprocher encore de Vaudelin, il faut toutefois faire à nouveau mouvement vers le Léon : déjà, le texte de 1800 était dû à un natif de Lanvéoc, sur la rade de Brest; plus proche encore, celui de 1730, dû au Léonard Charles Le Bris.

Restent les Pater de 1817 et 1775, qui arrivent en tête. Imprimés à Saint-Brieuc et à Morlaix pour le compte de l'évêché de Tréguier, ils n'ont pas, a priori, le profil de textes léonards. En effet, bien plus que les textes quimpérois, ils présentent de nombreuses adaptations à la prononciation locale, du nord-est de la Basse-Bretagne (par exemple : dimp pour deomp, «à nous »; beèt pour bezet, «soit»; oar pour var, «sur»; hirie pour hirio, «aujourd'hui »; reit pour roit, «donnez»; hon pour hor, «notre»; offanso pour offansou, «offenses»). Mais ces particularités restent superficielles et ne modifient pas les textes en profondeur.

Il existe pourtant bien au XVIII ${ }^{\mathrm{e}}$ siècle une tradition littéraire dans cette partie de la Bretagne, comme en atteste la transmission de manuscrits (dont ceux de Jean Cadec et Jean Conan sont les exemples les plus remarquables). En outre, l’imprimerie y fut

83. Even, 1987, p. 114.

84. Qu'on aurait pu s'attendre à voir mieux placé. Toutefois, le texte de 1691 (qui diffère peutêtre de ceux en usage dans le Léon auparavant) a servi à catéchiser des enfants nés à partir de 1697 seulement, qui avaient donc 18 ans en 1715. Il n'est pas étonnant alors que l'informateur de Vaudelin (dont on ne sait à quelle génération il appartenait) ait pu réciter un texte différent.

85. Ces deux Pater sont rigoureusement identiques (voir note suivante).

86. Le texte de 1800 a été imprimé à Prague par l'abbé Dumoulin, prêtre émigré, originaire de Lanvéoc et qui officia à Ergué. Le catéchisme de 1814 est la réédition du catéchisme prérévolutionnaire. Le Pater qu'il contenait a été recyclé tel quel, sans la moindre retouche, dans le catéchisme napoléonien de 1807. 
introduite précocement, puisque le Catholicon vit le jour à Tréguier en 1499. Mais cette activité fut très réduite par la suite : selon Gautier ${ }^{87}$, Pierre Levieil, imprimeur avec privilège épiscopal, y travailla de 1723 à 1762. Mais il n'est pas certain qu'il y en ait eu le moindre entre les $\mathrm{XV}^{\mathrm{e}}$ et $\mathrm{XVIII}^{\mathrm{e}}$ siècles. Quant à la production de Levieil, dont on ne retrouve guère de traces, on ne peut pas dire qu'elle ait ni abondé ni tenu compte des besoins d'un lectorat local, qui était probablement très réduit. Il semble donc que la tradition trégoroise d'écriture ait surtout concerné les laïcs et très peu l'Église, gagnée au prestige de la production livresque léonarde. Les premiers livres imprimés en breton à connaître une large diffusion dans l'évêché de Tréguier furent ainsi vraisemblablement les trois catéchismes antérieurs à la Révolution : en 1769, celui de Mgr de Royère; en 1775, celui de Mgr de Frétat de Sarra; et, en 1783, celui de Mgr Le Mintier, dont le catéchisme de 1817 est la réédition ${ }^{88}$. Il ne faut donc pas s'étonner d'y retrouver une simple transposition dialectalisée des formules léonardes, nettement mieux assises : on a en fait des textes «trégorisés » pour la forme, mais léonards/littéraires quant au fond. Et ce sont donc ces textes qui offriraient le meilleur reflet des formules rituelles courantes dans la moitié nord-ouest de la Basse-Bretagne du XVIII siècle.

\section{Conclusions}

Comme Cohen ${ }^{89}$ l'avait pressenti pour les germanistes, au celtisant non plus, le Pater de Vaudelin n’apprendra pas grand-chose qu'il ne sache déjà. Du moins en ce qui concerne les caractéristiques internes de la langue. Il s'agit d'un Pater de coloration très léonarde, enfant de son temps et de la région, assez vaste, qui l'a vu naître.

Pourtant, il s'agit bien d'un témoignage très précieux, tant par l'exceptionnalité de sa formule introductive que par l'éclairage qu'il apporte sur l'histoire externe de la langue. En l'état du corpus rassemblé, il ne paraît guère possible de le rattacher à une source précise. Mais ainsi apparaît une des grandes particularités de la société bretonnante, société de l'oralité. On peut faire l'hypothèse que tant de versions différentes du Pater ont circulé qu'inévitablement, chacune a laissé une trace quelque part, dans la mémoire d'une paroisse ou ne serait-ce que d'un locuteur. Et ce, d'autant plus facilement qu'elles étaient transmises oralement à une population massivement analphabète, hermétique à l'idée d'une forme de référence, qui forcément recomposait au gré des années, des générations ou des personnes, un texte qui devait toujours sembler le même par-delà la variation. À en juger par cette recommandation de C. Marigo sur la façon de réciter ses prières, l'Église elle-même n'était d'ailleurs peutêtre pas si regardante non plus sur les formes : Ne d'e quet ret o gouzout guer evit guer,

87. Gautier, 1857, p. 24.

88. Le Mintier, dernier évêque du siège de Tréguier, fut lui aussi un tenant de la tradition catholique et un ferme opposant à la Révolution. En septembre 1789, il adressa un mandement exhortant les fidèles à prier pour le rétablissement de l'ordre dans le royaume. Il émigra en 1791 et mourut en Angleterre.

89. Cohen, 1946, p. 2. 
avoalc'h e gouzout ar sustanç anezo ${ }^{90}$. Il était bien suffisant que les clercs détinssent la version de référence à travers le latin, éventuellement le français, quand le breton, «langue pauvre et stérile» (dixit Charles Le Bris ${ }^{91}$ ), ne servait que d'approximation à l'usage du peuple.

Malgré cela, il est certain que l'écrit a joué un rôle dans la composition du texte de Vaudelin. C'est indéniable si l'on se réfère aux blancs typographiques, voire éventuellement à la coupe étymologique de en Neñv, «aux Cieux », ainsi qu'à certaines liaisons, qui ne sont pas faites conformément à la phonétique mais seulement à la tradition graphique. Quel écrit? Voilà qui est impossible à déterminer. Y a-t-il eu plusieurs informateurs, qui auraient produit des versions concurrentes, arrangées tant bien que mal par Vaudelin? C'est une possibilité. Le ou les informateurs ont-ils mélangé le texte du Pater avec d'autres prières? Encore une possibilité, tout aussi invérifiable...

Il reste qu'avec ces interrogations, ce pauvre petit fragment égaré, notant fidèlement le breton parlé en 1715, recèle indéniablement pour le linguiste le même pouvoir de fascination qu'une étoile dont on voit encore la lumière trois siècles après sa disparition.

Erwan LE PIPEC

Université de Bretagne Occidentale erwan.lepipec@univ-brest.fr

90. «Il n'est pas besoin de les savoir mot-à-mot, il suffit d'en savoir la substance» : MARIGO, 1835 [1756], p. 422.

91. Cité par Roudaut, 1997, p. 234. 


\section{BIBLIOGRAPHIE}

\section{ABRÉVIATIONS}

ALBB : Le Roux, Pierre, Atlas linguistique de la Basse-Bretagne, Rennes/Paris, Plihon et Hommay / Champion, 1924-1963. Nouv. éd. Brest, Éditions armoricaines, 1977.

NALBB : Le Dû, Jean (dir.), Nouvel atlas linguistique de la Basse-Bretagne, Brest, CRBC, 2001 .

\section{SOURCES}

Euvres anonymes

Catechis an escopty a Guemper (catéchisme de l'évêché de Quimper), Quimper, 1814.

Catechis evit an oll ilizou emeus an Impalaërder a Franç (catéchisme de l'évêché de Quimper), Quimper, Barazer, 1807.

Catechis imprimet dre urs an Autro [...] Escop ha Cont a Dreguer (catéchisme de l'évêché de Tréguier), Morlaix, Guyon, 1775.

Catechis (catéchisme de l'évêché de Saint-Brieuc), Saint-Brieuc, Prud'homme, 1817.

Cantiquou spirituel, éd. Le Menn, Saint-Brieuc, Skol, 1997.

Bellarmin, trad. Gueguen, 1625 : Bellarmin, Robert, Declaration abundant eves an Catechism hac an doctrin Christen, trad. Euzen Gueguen, Morlaix, Allienne, 1625.

Chamberlayne, 1669 : Chamberlayne, Edward, Anglice notitia, or The present state of England together with divers reflections upon the antient state thereof, [Londres], John Martyn, 1669.

Des Rues, 1610 : Des Rues, François, Description contenant toutes les singularitez des plus célèbres villes et places remarquables du Royaume de France, Rouen, David Geoffroy, 1610.

Dumoulin, 1800 : Dumoulin, Alain, Grammatica latino-celtica, Prague, [s. 1.], 1800.

Fleury, trad. Dréano, 1851 : Catechim historik en abad Fleuri, laqueil é breton dré en eutru Dreano..., Guéned, Lamarzelle, 1851.

Gessner, 1555 : Gessner, Conrad, Mithridates, De differentiis linguarum tum veterum tum quae hodie apud diversas nationes in toto orbe terrarum in usu sunt, Zurich, Froschauer, 1555; en ligne : http://gallica.bnf.fr/ark:/12148/bpt6k938671.

Gueguen, éd. Hemon, 1977 : Gueguen, Tanguy, Doctrin an christenien, éd. Roparz Hemon, Dublin, The Dublin Institute for Advanced Studies, 1977.

Kerampuilh, éd. Stokes, 1876 : Kerampuilh (de), Gilles, Middle-Breton Hours, éd. Whitley Stokes, Calcutta, [s. l.], 1876.

Le Bris, [v. 1710] : Le Bris, Charles, Introduction d'ar Vuez Devot, Quimper, Derrien, [s. d.].

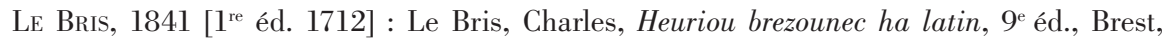
Lefournier, 1841.

LE BRIs, [v. 1730] : Le Bris, Charles, Instruction var an Excellanç, ar Froez, an Indulgeançou bras hac an Deveryou ar Vreuriez ar Rosera, Quimper, Derrien, [s. d.].

Lотн, 1890 : Loth, Joseph, Chrestomathie bretonne, Paris, Bouillon, 1890. 
Lотн, 1904 : Loth, Joseph, «Le plus ancien texte suivi en breton de Vannes», Annales de Bretagne, 20, 3, 1904, p. 341-350.

Marigo, 1835 [1 $1^{\text {re }}$ éd. 1756] : Marigo, Claude, Imitation hor Salver Jesus-Christ, $5^{\mathrm{e}}$ éd., Quimper, Blot, 1835.

Marion, 1790 : Marion, Jean, Instructioneu santél ar er gurionnéeu principal ag er religion, Vannes, Galles, 1790.

MaunorR, [v. 1646] : Canticou spirituel hac instructionou profitabl, Quimper, Perier, [s. d.]

Maunorr, 1659 : Maunoir, Julien, Le Sacré Collège de Jésus, Quimper, Hardouyn, 1659.

Maunoir, 1707-1732 [1 $1^{\text {re } e ́ d . ~ v . ~ 1660] ~: ~ M a u n o i r, ~ J u l i e n, ~ A n ~ A b r e g e ́ ~ e u s ~ a n ~ d o c t r i n ~ c h r i s t e n, ~}$

Quimper, Périer, éd. posthume 1707-1732.

Merula, 1607 : Merula, Paul, Cosmographiae generalis libri tres, Amsterdam, Guillaume Blaev, 1607; $1^{\text {re }}$ éd. Amsterdam, Cornelius Nicolas, 1605.

QuiQuer, 1626 : Quiquer, Guillaume, Dictionnaire et colloques françois et breton, Morlaix, Allienne, 1626.

QuiQuer, 1632 : Quicquer, Guillaume, Dictionnaire et colloques françois, breton et latin, Morlaix, Allienne, 1632.

QuiQuer, 1633 : Quiquer, Guillaume, Seconde partie du Dictionnaire et colloques françois, breton, [s. 1.], 1633.

Roparz, 1743 : Roparz, Euzen, An Imitation Jesus-Christ hon salver biniguet, 1743, Quimper, Périer, 1743.

Salmon, trad. Jurain, 1776 [1768] : Salmon, Thomas, Description générale de l'univers traduite de l'anglois de Salmons d'après la XVédition donnée à Londres en 1768, trad. abbé Jurain, Paris, Frouillé, 1776.

VAudeLin, 1713 : Vaudelin, Gile, Nouvelle manière d'écrire comme on parle en France, Paris, Jean Cot, 1713.

VaudeLin, 1715 : Vaudelin, Gile, Instructions crétiennes, mises en ortografe naturelle, pour faciliter au peuple la lecture de la science du Salut, Paris, Lamesle, 1715.

\section{ÉTUDES}

Adelung, 1809 : Adelung, Johann Christoph, Mithridates oder allgemeine Sprachkunde, Berlin, Vossischen Buchhandlung, II, 1809.

Bisquerra, 2009, Bisquerra, Claude, «Les catéchismes en plusieurs langues dans l'Ancien Régime », Documents pour l'histoire du français langue étrangère ou seconde, 43, 2009, p. 41-58.

Brunot, 1966 : Brunot, Ferdinand (dir.), Histoire de la langue française, Paris, A. Colin, VI, 1966.

Chaurand, 1999 : Chaurand, Jacques (dir.), Nouvelle histoire de la langue française, Paris, Seuil, 1999.

Cohen, 1946 : Cohen, Marcel, Le Français en 1700 d'après le témoignage de Gile Vaudelin, Paris, Champion, 1946.

Even, 1987 : Even, Arzel, Istor ar yezhoù keltiek, Lesneven, Hor Yezh, 2éd. 1987.

FAGON, 2004 : Fagon, Christian, «Changement du /z/ en /h/ en Léon : continuité du britto- 
nique au breton moderne et regard sociolinguistique des locuteurs sur ce phénomène », $L a$ Bretagne linguistique, 13, 2004, p. 73-83.

Favereau, 1997 : Favereau, Francis, Grammaire du breton contemporain, Morlaix, Skol Vreizh, 1997.

Gautier, 1857 : Gautier, Toussaint, Histoire de l'imprimerie en Bretagne, Rennes, Péalat, 1857. JACKson, 1967 : Jackson, Kenneth H., A Historical Phonology of Breton, Dublin, The Dublin Institute for Advanced Studies, 1967.

Le Berre, 2012 : Le Berre, Yves, Entre le riche et le pauvre, Brest, Emgleo Breiz, 2012.

LE Bihan, 2010 : Le Bihan, Herve, «Ar skridoù krennvrezhoneg en oberenn Seán Ó Tuathalláin», Hor Yezh, 262, 2010, p. 39-42.

Martinet, 1969 : Martinet, André, Le Français sans fard, Paris, PUF, 1969.

Roudaut, 1997 : Roudaut, Fañch, « La littérature religieuse en breton », in Fleuriot, L., Ségalen,

A.-P. (dir.), Histoire littéraire et culturelle de la Bretagne, I. Héritage celtique et captation française, Paris/Spézet, Champion/Coop Breizh, 1997, p. 231-243.

Walter, 1976 : Walter, Henriette, La Dynamique des phonèmes dans le lexique français contemporain, Paris, Droz, 1976.

Widmer, 2012 : Widmer, Paul, «A so and so in Middle and Early Modern Breton : A quantitative approach », Hor Yezh, 270, 2012, p. 31-39. 